\title{
Medición de presión arterial con Doppler en recién nacidos y lactantes normales
}

\author{
Dra, Edda Lagomarsino F., 2 ; Dra. Bettina von Dessauer G.' ; Dra. Helia Molina M.', 2 ; \\ Dr. Eric Solar G. ${ }^{2}$; Sr. Rodrigo Gajardo L. ${ }^{3}$
}

Blood pressure by Doppler method in 0 to 24 months old infants.

\begin{abstract}
Systolic blood pressure (SBP) was measured by Doppier method in an aleatory sample of 251 healthy children from south-east Santiago Chile (131 females and 120 males) which were divided by age in five groups: 0 to 28 days $(n=5) 1$ to 5 months $(n=48), 6$ to 11 months $(n=48), 12$ to 17 months $(n=46)$ and 18 to 24 months $(n=45)$. Great dispersion of SBP values occurred among newborn infants (range 85 to 125 ) in comparisson with groups 1 to 5 months (105 to 130$), 6$ to 11 months (118 to 130). Fifty and 95 percentiles were, respectively, 107 to 125 ; 116 to $130 ; 121$ to $130 ; 118$ to 130 and 120 to 130 . These values were greater than those obtained by sphygmomanometry in a similar matched group of children.
\end{abstract}

(Key words: blood pressure, hypertension, Doppler technique.)

En 1957 Satomura ${ }^{1}$ aplicó el principio de] Doppler para el diagnóstico de anormalidades cardiovasculares. Kemmerer ${ }^{2}$ encuentra, en 1967, que la presión arterial sistólica obtenida por el Doppler se correlaciona bien con las medi-

1. Departamento de Pediatr ŕa, Universidad Católíca.

2. Servicio Nefrología, Hospital Sótero del Río.

3. Ingeniero Comercial, Cátedra Informática Estadística Facultad de Ciencias Económicas y Administrativas, Universidad de Chile. ciones directas. El uso pediátrico de la técnica del Doppler fue sugerido, en 1968, por Stegall ${ }^{3}$.

La técnica está basada en el principio de Doppler, esto es, el cambio de frecuencia que experimenta una onda de ultrasonido a ser reflejada por los corpúsculos que se desplazan dentro del vaso sanguíneo 4,5 .

El propósito đe este estudio es establecer, con este método, el rango de presiones sistólicas de recién nacidos y lactantes normales con sus percentiles, p5, p50 y p95 y compararlas con las 
obtenidas con el esfigmomanómetro. No existen publicaciones nacionales sobre medición de pre. sión, con esta metódica, en recién nacidos.

\section{MATERIAL Y METODO}

Se procectió a medir la presión arterial sistólica utiji. zando un equipo Doppler Sonicaid BV 102 R/BV 102, que opera con una frecuencia de $8 \mathrm{MHz}$, con transductor de $15 \mathrm{mw} / \mathrm{cm}^{2}$, a una muestra aleatoria de 251 nitios de ambos sexos: 131 mujeres y 120 hombres con edades que fluctuaban entre 0 a 24 meses de vida, divididos en 5 grupos con un promedio de 50 niños por grupo de 0 a 28 días (RN), 1 a 5,6 a 11,12 a 17 y 18 a 24 meses. (Tabla 1).

El procedimiento lo realizó un solo operador en la maternidad, sala cuna y policlínion de niño sano del Servicio de Salud Metropolitano Sur Oriente (SSMSO). Los niños estaban on condiciones basales: en decúbito, despiertos, tranquilos, una a dos horas después de alimentados. Se utilizó brazaletes que ocupaban los dos tercios del brazo derecho, sobre la arteria humeral. EI manguito se infló a $20 \mathrm{mmHg}$. sobre el punto en que el sonido cesaba y se bajó a razón de 2 a $3 \mathrm{~mm}$ por segundo. Presión sistólica fue el punto en que el sonido reapa. recía ${ }^{3}$. Se tomaron tres registros de cada niño, cuyos resultados se promediaron. Fueron descartados los recién nacidos prematuros, los niños enfermos y aqueLos a quienes no fue posible tranquilizar. La clasificación uutricional fue comparable a la de la población pediátrica menor de dos arios del SSMSO*.

El análisis estadístico se realizó calculándose tas medias y desviaciones estándar para la muestra como un todo, para cada corte etario y por sexo. Se graficó la frecuencia de presiones por grupo y se confecciono una nube de puntos para relacionar presiones $y$ edades para cada niño. Se calculó para todo el grupo los percentiles p5, p50 (o mediana) y p95. Se compararon las medias obtenidas para todo el gupo con las obtenidas utilizando el esfigmomanómetro de mercurio en todos los niños mayores de un año $y$ aquellos menores de un afto en que el sonido arterial fue perceptible.

Tabla 1

Distribución de la población estuđiada con Doppler por edad y sexo

\begin{tabular}{lccccc}
\hline \multirow{2}{*}{$\begin{array}{l}\text { Edad } \\
\text { meses }\end{array}$} & \multirow{2}{*}{$N^{\circ}$ de casos } & \multicolumn{2}{c}{ Hombre } & \multicolumn{2}{c}{ Mujer } \\
& & N & \% & \multicolumn{1}{c}{ N } & $\%$ \\
\hline $0-0,99$ & 50 & 27 & 54 & 23 & 46 \\
$1-5,99$ & 48 & 21 & 43,8 & 27 & 56,3 \\
$6-11,99$ & 48 & 22 & 45,8 & 26 & 54,2 \\
$12-17,99$ & 46 & 16 & 34,8 & 30 & 65,2 \\
$18-23,99$ & 45 & 25 & 55,5 & 20 & 44,4 \\
Total & 237 & 111 & 100 & 126 & 100 \\
\hline
\end{tabular}

\section{RESULTADOS}

El promedio y rango de presión arterial sistólica del grupo estudiado separados por edad y sexo se presentan en la tabla 2 . Los valores son similares para ambos sexos, con un rango amplio en cada grupo. Se observa un ascenso del valor promedio de $107 \mathrm{mmHg}$ en los recién nacidos, en ambos sexos, y a 119 y $122 \mathrm{mmHg}$ en los lactantes varones y mujeres, respectivamente, entre 18 y 24 meses.

Los coeficientes de correlación obtenidos no tuvieron significación estadística. Para el grupo hombres fue 0,32 y mujeres 0,41 . Ambas pendientes fueron positivas.

En la figura 1 se relaciona edad y presión arterial de cada individuo. Se observa un alta dispersión en todos los grupos, mayor en los recién nacidos.

\section{Tabla 2}

Promedio y rango de presión arterial con Doppler ел 251 niños de 0-24 meses separados por sexo $y$ edad

\begin{tabular}{crrrr}
\hline & \multicolumn{4}{c}{ Presín arterial (mmHg) } \\
Edades & $\begin{array}{c}\overline{\mathrm{X}} \\
\text { (meses) }\end{array}$ & Rango & $\overline{\mathrm{X}}$ & Rango \\
Hombre & & Mujer & \\
\hline $0.0,99$ & 107,4 & $\mathbf{8 5}-125$ & 107,2 & $\mathbf{8 5 - 1 2 0}$ \\
$1-5,99$ & 119,1 & $110-130$ & 118,2 & $\mathbf{1 0 5 - 1 3 0}$ \\
$6-11,99$ & 118,6 & $110-130$ & 120,8 & $110-130$ \\
$12-17,99$ & 120,0 & $105-130$ & 120,0 & $110-130$ \\
$18-23,99$ & 119,2 & $110-130$ & 122,0 & $115-130$ \\
\hline
\end{tabular}

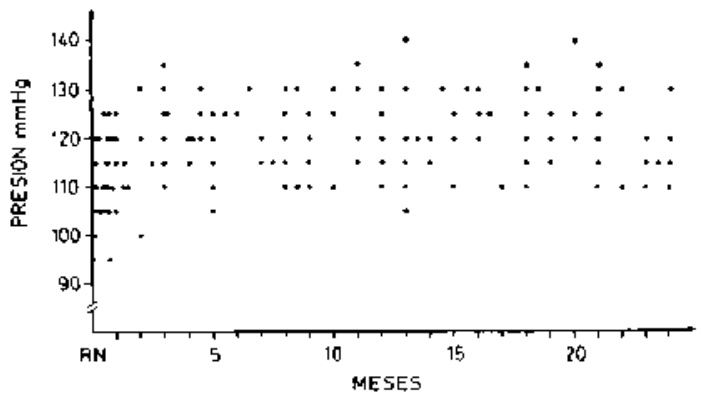

Figura 1: Nube de puntos de presiones relacionados con edades para niflos desde recién racidos a 24 meses de edad. 
En la figura 2 se presenta la distribución de frecuencia de los 5 grupos. En los recién nacidos la mayor frecuencia estuvo en el rango de 110 a $115 \mathrm{mmHg}$; de 1 a 5 meses entre 115 a 125 $\mathrm{mmHg}$; de 6 a 11 meses entre 120 a $130 \mathrm{mmHg}$; de 12 a 24 meses entre 120 a $125 \mathrm{mmHg}$.

En la figura 3 se muestra la curva obtenida con el cálculo de los percentiles 5 a 50 (mediana) y 95. Las medianas fueron $107 \mathrm{mmHg}$ para los recién nacidos; $116 \mathrm{mmHg}$ para el grupo 1 a 5 meses; $121 \mathrm{mmHg}$ para 6 a 11 meses; $118 \mathrm{mmHg}$ para el de 12 a 17 y $120 \mathrm{mmHg}$ para el entre 18 y 24 meses.

El percentil 95 en nuestro estudio fue de $124,58 \mathrm{mmHg}$ para los recién nacidos y 133,33 $\mathrm{mmHg}$ para los cuatro grupos restantes.

En la figura 4, al comparar las curvas de presión obtenidas con Doppler y las obtenidas con el esfigmomanómetro de mercurio se observa que los promedios con ambas técnicas tienen tendencias positivas, a medida que aumenta la edad, aunque la medición con Doppler muestra resultados que están fuera de la extrapolación de la mediciôn con esfigmomanómetro.
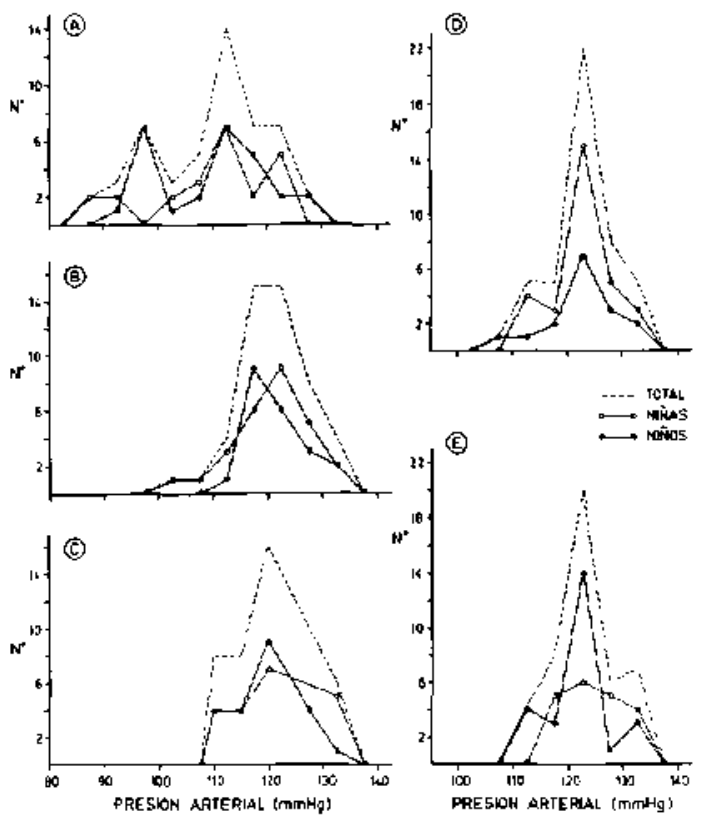

Figma 2: Distribución de frecuencias de presión sistólica con Doppler en nit̂os menores de 2 años. A: en recién nacidos; B: de 1 a 5 meses; C; de 6 a 11 meses; D: de 12 a 17 meses, y E: de 18 a 24 meses.

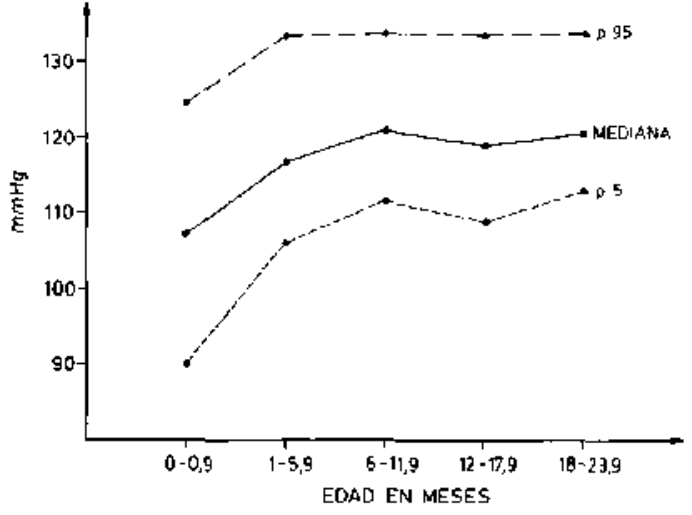

Figura 3: Presión sistólica con Doppler en nî̃os de 0 a 24 meses de edad. Se calculó el p5, p50 o mediana y p95.

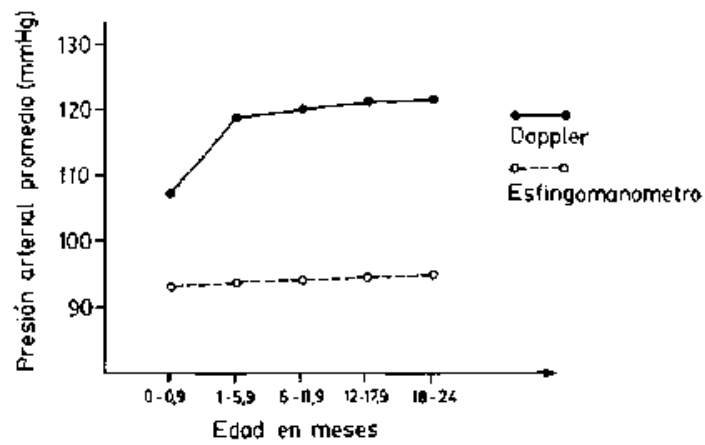

Figura 4: Correlación entre presión sistólica promedio y edad tomadas con Doppler y esfigmomanómetro de mercurio.

\section{COMENTARIO}

La necesidad de tomar la presión arterial a recién nacidos y lactantes se debe a la frecuencia cada vez mayor de la hipertensión arterial en estas edades?. Sin embargo, la existencia de diversos métodos para determinar las cifras tensionales destacan la dificultađ de obtener cifras confiables y comparables, por lo que se desconocen los valores sospechosos sobre los cuales trabajar.

Los métodos habituales para medir la presión en los recién nacidos y lactantes menores inclu. yen la auscultación, la palpación, el enrojecimien. to (Flush), la medición directa y la técnica del 
ultrasonido o Doppler. El método auscultatorio está a menudo difícultado en el niño pequeño por lo suave $y$ a veces imperceptible del sonido arterial. El método palpatorio, usando la arteria braquial, es simple y se emplea frecuentemente, mide presión sistólica $y$, generalmente, lee 5 a 10 mmHg bajo la presión sist 6 tica real ${ }^{8}$.

El método del enrojecimiento cada vez se usa menos. Es una técnica económica, pero necesita dos operadores y es insegura. Grupos experimentados tienen divergencias en la lectura del resultado $^{9}$. Mide la presión media, no identifica los niveles sistólicos o diastólicos, los valores obtenidos son influidos por la anemia, edema o hipoter$\mathrm{mia}^{8}$. La medición directa de la presión arterial es exacta y reproductible. Es el estándar con el cual los otros métodos son evaluados. Se obtiene empleando un transductor de presión unido a un catéter que ha sioo instalado en la aorta vía arteria umbilical. Es una técnica invasiva e implica riesgo de complicaciones. La técnica del ultrasonido del Doppler es simple, no invasiva y está siendo usada cada vez más en la medición de la presión de los recién nacidos y lactantes pequeños. Los valores de la presión sistólica obtenidos por el Doppler se correlacionan bien con aquellos medidos directamente intraaórticos ${ }^{10-12}$.

Además de la técnica usada hay muchas otras variables que afectan las lecturas de la presión arterial del recién nacido y lactante. Estas incluyen el peso y la edad gestacional ${ }^{13,14}$, el estado de vigilia ${ }^{15}$, la alimentación previa y el tamaño del manguito usado ${ }^{16}$. El llanto aumenta las cifras tensional promedio en $18 \mathrm{mmHg}^{17}$.

Al correlacionar en nuestro estudio las presiones con edades se aprecia una alta dispersión determinada por el bajo coeficiente de correlación.

La forma de la distribución de frecuencia tiende hacia una curva normal de Gauss cada vez más concentrada a medida que aumenta la edad.

El percentil 95 en nuestro estudio fue 124,5 $\mathrm{mmHg}$ para recién nacjdos y de $133,3 \mathrm{mmHg}$ para los cuatro grupos restantes. Los niños con estas cifras o cifras superiores deberían considerarse hipertensos. Las medidas obtenidas con Doppler son promedio $20 \mathrm{mmHg}$ superiores a las obtenidas por los autores con esfigmomanómetro. Hecho explicable, porque el Doppler detecta la presión de flujo, es decir, el momento en que al desinflarse el manguito comienza a fluir la corrjente de glóbulos rojos mientras el esfigmomanómetro detecta el momento en que el flujo es suficiente para movilizar la pared arterial.
Al comparar nuestro estudio $y$ el de Brompton ${ }^{18}$, en que se siguió la misma técnica, la forma de las curvas es igual, pero las nuestras se situaron en cifras mayores, lo que puede explicarse porque los otros autores midieron las presiones en el hogar del niño. Si las comparamos con las cifras entregadas por la Segunda "Task Force" 19 , éstas son iguales a las obtenidas por nosotros con esfigmomanómetro. En el espacio que la "Task Force" le dedica a presión en RN y lactantes no especifica la técnica usada, ya que en las figuras aparecen presiones sistólicas y dias. tólicas y en la tabla sólo sistólicas. Nuestros resultados son comparables a los obtenidos en lactantes de 2 a 3 meses de edad en un consultorio chileno'.

En síntesis, se puede concluir que las cifras de presión arterial son más variables mientras menor es la edad del niño. Existe baja relación entre edad y presión arterial en el grupo etario de 0 a 24 meses. La presión arterial promedio fue en aumento con la edad, pero a tasas decrecientes. La forma de distribución de frecuencia de presión arterial tiende hacia una curva normal, de Gauss, cada vez más concentrada a medida que aumenta la edad. Existe poca relación entre presiones obtenidas con Doppler y las obtenidas con el esfigmomanómetro. Las medidas con Doppler son más altas.

\section{RESUMEN}

Se midió la presión arterial con la técnica del Doppler a una muestra aleatoria de 251 niños sanos: 131 mujeres y 120 hombres, divididos en 5 grupos de 0 a $28(n=50)$ días; 1 a $5(n=48)$; 6 a $11(n=48) ; 12$ a $17(n=46)$ y 18 a 24 $(n=45)$ meses de edad del área sur oriente de Santiago.

En RN existe una gran dispersión; la mayor frecuencia de presiones estuvo en el rango 85 a $115 \mathrm{mmHg}$; en los lactantes de 1 a 5 meses entre 115 a $125 \mathrm{mmHg}$; en los de 6 a 11 meses entre 120 a $130 \mathrm{mmHg}$, y en los con 12 a 24 meses entre $120-125 \mathrm{mmHg}$. El percentil 50 fue de $107 \mathrm{mmHg}$ para $\mathrm{RN} ; 116 \mathrm{mmHg}$ para el grupo de 1 a 5 meses; $121 \mathrm{mmHg}$ para el de 6 a 11 meses; $118 \mathrm{mmHg}$ para el de 12 a 17 meses, y $120 \mathrm{mmHg}$ para el de 18 a 24 meses. El percentil 95 fue de 125,5 para $\mathrm{RN}$ y $133,3 \mathrm{mmHg}$ para los otros grupos. Estas cifras son mayores que lo obtenido con esfigmomanómetro. 


\section{REFERENCIAS}

1. Satomura $S_{\text {. }}$ Ultrasonic Dopplet method for the inspection of cardiac functions. Acoust Soc Am 1957; 29:1181-1187.

2. Kemmerer WT., Ware $R W$., Stegall H., Evans WE. Indirect measurement of human blood pressure by the Doppler ultrasonic technique, Surg Forum 1967; 18:163-166.

3. Stegoll $H F$. Kardon MB., Kemmerer WT.: Indirect meassurement of arterial blood pressude by Doppler ultrasonic sphingomanometry. J Appl Physiol $1968 ; 25: 793-798$.

4. Yas JST: Arterial survey with the Doppler U1trasonic velocity detector en Rutherford $\mathbf{R}$. Vascular Surgery, USA, W.B. Saunders Company $1977,87.95$.

5. Kirkland RT, Kirkland $I L .:$ Systolic blood pressure measurement in the newborn infant with the transcutaneous Doppler method J Pediatr 1972; 80: 52-56.

6. Puentes $R$.: Desnutrición Infantil en Winter A., Puentes R. eds. Manual de Pediatría, Chile J.M.C., Color, Edic. 1984; 446-466.

7. Adleman RO.: The epidemiology of neonatal hypertension en Gioyannelli G., eds.: Hypertension in children and adolescents. New York; Raven Ptess; 1981 ; 21-29.

8. Moss $A J$ : Indirect methods of blood pressure measurement. Pediatr Clin North Am 1978; 25: 3-14.

9. Scroggie AS., Gallardo GE., Montenegro HD y col.: Medición simultánea indirecta de la presión arterial en lactantes mediante tres métodos. Rev Chil Pediatr 1982; 3: 558-561.
10. De Swett M., Fayer P., Shineboums EA.: Blood pressure survey in a population of newborn infants, Brit Mad 3 1976; 2:9-11.

11. Dwech HS., Reynalds $D W$., Cassady $G_{\text {: }}$ : Indirect blood pressure measurement in newborns. Am J Dis Child 1974; 128: 492-498.

12. Hernández A., Goldring D., Hortmon AE. Measurement of blood pressure in infant and children by the Doppler uitrasound technique Pediatrics 1971; 48: $788-801$.

13. Swyer $P R$.: Blood pressure in normal fult term and premature infants. Am J Dis Child 1986; 113: 374 . 377.

14. Whari M.: Changes in blood pressure during the first year of life. Acta. Pediatr Scand 1980; 69: 613-617.

15. Lee YH., Rosner B., Gould JB., Lowe EW. Kass EM: Familial aggregation of blood pressure of newborn infants and the mothers. Pediatrics 1976; 58: 722-729.

16. Moss $A J$., Admas $F H$.: Auscultatory and intra-arterial pressure; A comparison in children with special references to cuff width J Pediatr 1965; 66: 10941097.

17. Moss AJ., Duffie ER., Emmanouilides GC.: Blood pressure and vasomotor reflexs in the newborn infants. Pediatrics 1963; 32: 175-179.

18. De Swiet $M$., Fayers $P$., Shineboums EA.: Systolic blood pressure in a population of infants in the first year of life. The Brompton study. Pediatrics 1980;8: 1028-1035.

19. Report of the Second Task Force on Blood Pressure Control in Children 1987. Peđtiatrics 1987; 79: 1-25. 